\title{
Blockade of KCa3.1 Attenuates Left Ventricular Remodeling after Experimental Myocardial Infarction
}

\author{
Chen-Hui Ju ${ }^{\mathrm{a}}$ Xian-Pei Wang ${ }^{\mathrm{a}}$ Chuan-Yu Gao ${ }^{\mathrm{a}, \mathrm{b}}$ Shuang-Xia Zhang ${ }^{\mathrm{a}}$ \\ Xing-Hua Ma ${ }^{a}$ Chang Liu ${ }^{\mathrm{a}}$
}

aDepartment of Cardiology, Zhengzhou University People's Hospital, 'benan Institute of Cardiovascular Epidemiology, Zhengzhou, China

\section{Key Words}

Myocardial infarction $• \mathrm{KCa} 3.1 \cdot$ Fibroblasts $\cdot$ TRAM-34 • Ventricular remodeling

\begin{abstract}
Background/Aims: After myocardial infarction (MI), cardiac fibrosis greatly contributes to left ventricular remodeling and heart failure. The intermediate-conductance calcium-activated potassium Channel (KCa3.1) has been recently proposed as an attractive target of fibrosis. The present study aimed to detect the effects of $\mathrm{KCa3.1}$ blockade on ventricular remodeling following MI and its potential mechanisms. Methods: Myocardial expression of KCa3.1 was initially measured in a mouse MI model by Western blot and real time-polymerase chain reaction. Then after treatment with TRAM-34, a highly selective KCa3.1 blocker, heart function and fibrosis were evaluated by echocardiography, histology and immunohistochemistry. Furthermore, the role of KCa3.1 in neonatal mouse cardiac fibroblasts (CFs) stimulated by angiotensin II (Ang II) was tested. Results: Myocardium expressed high level of KCa3.1 after MI. Pharmacological blockade of KCa3.1 channel improved heart function and reduced ventricular dilation and fibrosis. Besides, a lower prevalence of myofibroblasts was found in TRAM-34 treatment group. In vitro studies KCa3.1 was up regulated in CFs induced by Ang II and suppressed by its blocker.KCa3.1 pharmacological blockade attenuated CFs proliferation, differentiation and profibrogenic genes expression and may regulating through AKT and ERK1/2 pathways. Conclusion: Blockade of KCa3.1 is able to attenuate ventricular remodeling after MI through inhibiting the pro-fibrotic effects of CFs.
\end{abstract}




\section{Introduction}

Following myocardial infarction (MI), deaths in acute phase are prominently reduced due to timely revascularization treatment strategies, however, chronic heart failure remains a big threat to the living patients. Development of heart failure after MI is deeply associated with ventricular remodeling which is defined as profound changes in cardiac structure and function $[1,2]$. At the cellular and molecular level, fibroblast proliferation and collagen deposition in extracellular matrix (fibrosis) are the main characteristics of ventricular remodeling $[3,4]$. Currently approved drugs targeting the renin-angiotensin-aldosterone and sympathetic nervous systems exert its beneficial effects at least partially by regulating these processes [5]. However, the current therapeutic strategies cannot stop the progression of heart failure.

It is widely accepted that cardiac fibroblasts(CFs) are crucial in both cardiac repair and remodeling processes following MI. Moreover, after differentiation to myofibroblasts, it exhibit enhanced ability of migration, proliferation, and secretion, which are closely involved in adverse cardiac remodeling [6-8]. Increasing evidence suggests that ion channels regulate the functions of many cells. Potassium channels regulate calcium entry and calcium-signaling processes by maintaining a negative membrane potential, which is a key regulator in many cell processes $[9,10]$. KCa3.1 has been shown to play an important role in proliferation, migration, and activation of fibroblasts [11-13]. Additionally, selectively targeting KCa3.1 in fibroblasts ameliorates the progression of unilateral ureteral obstruction-induced renal fibrosis and may be a novel approach in treating idiopathic pulmonary fibrosis[11, 14].

The present study investigated the effects of KCa3.1 channel blockade on cardiac remodeling in a mouse $\mathrm{MI}$ model and potential mechanisms associated with CFs.

\section{Materials and Methods}

\section{MI and treatment}

Male C57BL/6 mice 8 to 10-week-old were purchased from Beijing University (Beijing, China). All experiments were carried out in compliance with the Guide for the Care and Use of Laboratory Animals published by United States National Institutes of Health and approved by the Institutional Authority for Laboratory Animal Care of China. MI was induced by permanent ligation of the left anterior descending(LAD) artery. Briefly, the mice were anesthetized with 3.3\% chloral hydrate intraperitoneally and mechanically ventilated through a rodent respirator. Between left third and fourth ribs thoracotomy was performed. The thymus was retracted upwards to expose the auricular appendix, and left lung was collapsed by a little sterile cotton to avoid injury. A 6-0 silk suture was used to ligate the LAD artery at a level approximately 1 $\mathrm{mm}$ below the edge of left auricular appendix. In the sham-operation group, the same procedure was done except ligating the LAD coronary artery. Mice were killed at 5days or 4 weeks following MI [15].

In the treatment group, mice were treated daily with TRAM-34 [Sigma-Aldrich Corporation, Missouri (MO), and United States of America (USA)] at $120 \mathrm{mg} / \mathrm{kg}$ by intraperitoneal injection starting 3 days before MI surgery. Control group received the same volume of vehicle peanut oil (Sigma-Aldrich Corporation, MO, and USA) alone.

\section{Histology and immunohistochemistry(IHC)}

At each set time point, mice were killed and hearts were rapidly excised. Then the hearts were fixed with $4 \%$ paraformaldehyde, paraffin embedded and cross-sectioned from base to apex into $1 \mathrm{~mm}$ thick sections. $7 \mu \mathrm{m}$ sections from each were used for additional study. Infarct size was measured by Masson's trichrome and calculated as the ratio of total infarct circumference to total LV circumference. Fibrosis was evaluated by Sirius red staining and presented as collagen content which calculated the percentage of interstitial fibrosis in non-infarct area. Image-Pro Plus software was used to analyze the images.

For the IHC staining, slides were deparaffinized and subjected to heat-mediated antigen retrieval. The anti- $\alpha$-smooth muscle actin(SMA, clone 1A4, 1;200, Abcam, Cambridge, and United Kingdom) and antiCD31[clone MEC13.3, 1:100, BD Biosciences, San Jose, California(CA), and USA]primary antibodies were 
Ju et al.: Blockade of KCa3.1 Attenuates Left Ventricular Remodeling

used followed by horseradish peroxidasebased staining and 3, 3'-diaminobenzidine revelation. Images ( $4 \sim$ to 5 per LV section) were acquired at 200 magnification in the border zone of the infarct area using a microscope and analysed using Image Pro Plus software. The results were expressed as numbers per $\mathrm{mm}^{2}$.

\section{Echocardiography}

At 0, 2, and 4 weeks after MI, mice were anesthetized and underwent transthoracic echocardiographic studies by a Vevo 2100 high-resolution micro imaging system (Visualsonic, Canada). At the level of papillary muscle M-mode tracings were recorded. Left ventricular end-systolic dimension (LVESd) and left ventricular end diastolic dimension (LVEDd) were measured. Fractional shortening (FS) was calculated. All data were averaged from five cardiac cycles.

\section{Western blotting}

Tissues or cells were lysed using a mixed lysis solution (Roche, Basel, and Switzerland). Protein concentrations were measured and samples were separated by a sodium dodecyl sulfate-poly acrylamide gel electrophoresis. After transferred to nitrocellulose membranes and blocked in 5\% dry milk, primary antibodies were used to incubate the proteins overnight at $4^{\circ} \mathrm{C}$. Anti KCa3.1 antibody was purchased from Alomone Laboratory (clone 6C1, 1:600, Jerusalem, Israel). Anti extracellular signal regulated kinase (ERK)1/2 (clone 137F5, 1;1000), phospho-ERK1/2 (clone 197G2, 1:1000), Akt (clone 11E7, 1:1000), and phospho-Akt(clone 244F9,1:1000) antibodies were obtained from Cell Signaling Technology(Beverly, Massachusetts, and USA). The membranes were washed three times and incubated with secondary antibody for $2 \mathrm{~h}$ at room temperature. Proteins were visualized and glyceraldehyde 3 phosphate dehydrogenase (GAPDH) was used as a control [16].

Quantitative real time-polymerase chain reaction (RT-PCR)

Total RNA was extracted from the myocardium or cultured fibroblasts with trizol reagent (Invitrogen Life Technologies, CA, and USA). The mRNA expression of target genes was quantified by RT-PCR using SYBR Green Master Mixture (Takara, Japan). The PCR primers sequences are listed in Table 1. The GAPDH housekeeping gene was used as a control. The relative expression quantity was calculated by $2^{-\Delta \Delta \mathrm{Ct}}$.

\section{Isolation and culture of neonatal mouse CFS}

Primary neonatal mouse CFs were isolated from 1-day-old C57Bl/6 mouse pups. Briefly, hearts were excised and washed in cold hanks balanced salt solution (HBSS) (Hyclone, Logan, Utah, and USA). The ventricles were dissociated with trypsin ( $1 \mathrm{mg} / \mathrm{ml}$, Gibco Life Technologies Inc., Grand Island, NY, and USA) at $4^{\circ} \mathrm{C}$ for $30 \mathrm{~min}$, then digested with collagenase (Invitrogen Life Technologies, CA, and USA) dissolved in $\mathrm{HBSS}$ at $37^{\circ} \mathrm{C}$ for $15 \mathrm{~min}$. After repeated digestion, supernatant containing cells were collected into dulbecco's modified eagle medium DMEM supplemented with 5\% fetal bovine serum (FBS). After centrifugation, the cell pellet was gently resuspended in DMEM/10\% FBS with a mixture of $100 \mathrm{U} / \mathrm{mL}$ penicillin/streptomycin (Invitrogen Life Technologies, CA, and USA) and incubated for $60 \mathrm{~min}$ at $37^{\circ} \mathrm{C}$. CFs readily attached to the bottom of the culture dish and cardiomyocytes enriched cell suspension was discarded. CFs were seeded

Table 1 Sequences of PCR primers

\begin{tabular}{|c|c|c|}
\hline & Forward primers & Reverse primers \\
\hline GAPDH & 5'-CGTCCCGTAGACAAAATGGTGAA-3' & 5'-GCCGTGAGTGGAGTCATACTGGAACA-3' \\
\hline KCa3.1 & 5'-CCTCCTTTGTCTTATTGTGGTC-3' & 5'-GGAAGCGGACTTGGTTGA-3' \\
\hline$\alpha-S M A$ & 5'-GGCACCACTGAACCCTAAGG-3' & 5'-ACAATACCAGTTGTACGTCCAGA -3' \\
\hline Procollagen I & 5'- GCTCCTCTTAGGGGCCACT -3' & 5'- CCACGTCTCACCATTGGGG -3' \\
\hline Procollagen III & 5'- ACGTAGATGAATTGGGATGCAG -3' & 5'- GGGTTGGGGCAGTCTAGTG -3' \\
\hline CTGF & 5'-GGGCCTCTTCTGCGATTTC -3' & 5'-ATCCAGGCAAGTGCATTGGTA-3' \\
\hline TGF- $\beta 1$ & 5'-CTCCCGTGGCTTCTAGTGC-3' & 5'-GCCTTAGTTTGGACAGGATCTG-3' \\
\hline
\end{tabular}


at $1 \times 10^{5}$ cells $/ \mathrm{cm}^{3}$ at the second passage on dishes or 96-well culture plates for study. Ang II(Enzo Life Sciences, Shanghai and China) was used as a stimulant. After serum starvation for $24 \mathrm{~h}$, CFs were incubated with serum free medium, Ang II $(0.1 \mu \mathrm{M})$ or Ang II $(0.1 \mu \mathrm{M})$ wih TRAM-34(0.1,1,and $3 \mu \mathrm{M})$ for 30 min or 24 h. TRAM-34 was added 30 min before Ang II . The concentrations of Ang II and TRAM-34 were referred elsewhere $[17,18]$.

\section{Cell proliferation measurements}

The measurement of cell proliferation was performed with the cell counting kit-8 (CCK-8) (Dojindo, Japan)per manufacturer's protocol. Briefly, at the set time point, the CCK-8 solution (10 $\mu \mathrm{L})$ was added into 96-well culture plates. After 2 hours incubation, the optical density (OD) value of each well at 450 $\mathrm{nm}$ wavelength was recorded. The relative cell number was calculated as the percentage of (OD test -OD blank)/(OD control - OD blank).

\section{Statistical analyses}

All data were expressed as the means \pm SEM. $\mathrm{P}<0.05$ was considered as statistically significant. The one-way analysis of variance test was used to compare variables among groups. Student's t-test was used to assess the statistical difference between two groups.

\section{Results}

Myocardial expression of KCa3.1

To test the involvement of KCa3.1 in MI, the study first investigated KCa3.1 expression levels in the infarct myocardium at different time points after MI. Its protein expression level increased significantly at day 1 . After a period of fluctuation, it raised again. In the later phase of MI, it reached to a higher level (Figs. 1A and 1B). The mRNA expression level peaked at day 3 and after a decrease it maintained at a high level (Fig. 1C). The mRNA trend was consistent with the protein.

Pharmacological blockade of KCa3.1 channels attenuated LV remodeling and cardiac dysfunction after MI

To identify the role of KCa3.1 in myocardial remodeling, mice treated with TRAM-34 or vehicle control were examined by ultrasound at 0,2 , and 4 weeks. Heart function was assessed by FS, while heart dilation was evaluated by LVEDd and LVESd. At 4 weeks, the TRAM-34 group had a significant improvement of heart function and reduction of $\mathrm{LV}$ dilation in comparison to vehicle group. This difference was not seen at 2 weeks (Fig. 2A-2B).

Infarct size, collagen content, prevalence of myofiroblasts, and microvessel density were assessed

Four weeks after MI, infarct size was measured by Masson's trichrome staining in TRAM-34 and vehicle groups and quantification analysis demonstrated no significant difference between them (Fig.3A). However, the treatment with TRAM-34 led to less interstitial fibrosis as assessed by collagen content (Fig. 3B) and decreased $\alpha$-SMA positive cells(Fig. 3C). As KCa3.1 had been reported to express in vascular cells, and also detected the density of microvessel but found no difference between two groups (Fig.3D). Thus, the infarct myocardium in TRAM-34 treatment group was demonstrated as decreased collagen content and lower prevalence of myofibroblasts.

KCa3.1 was up regulated in Ang II induced CFs and suppressed by TRAM-34

To explore the possible mechanisms involved in the beneficial effects of TRAM-34, the study investigated the role of KCa3.1 in neonatal mouse CFs. AngII, a major bioactive peptide of the renin angiotensin aldosterone system, plays a critical role in cardiac remodeling. The underlying mechanisms included stimulating CFs proliferation and collagen synthesis [19] .Thus, Ang II was used as a stimulator and found Ang II significantly increased the expression 
A

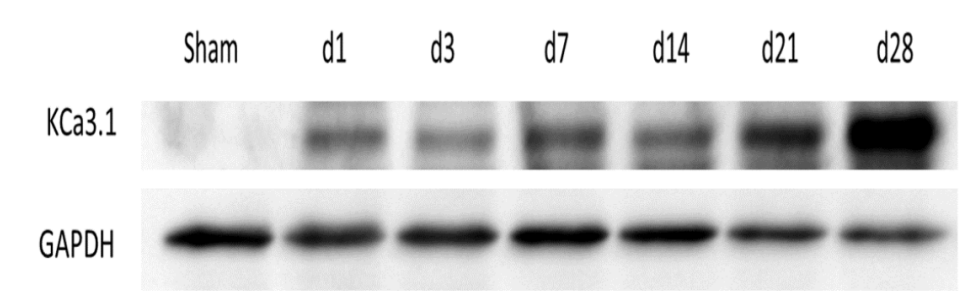

B

$\mathrm{C}$
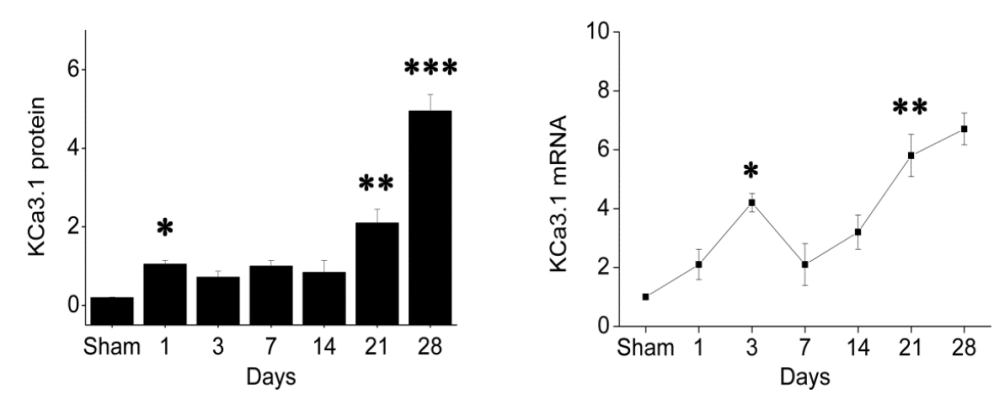

Fig. 1. Myocardium KCa3.1 expression after MI. A. Representative Western blot analysis of KCa3.1 channel protein expression at different times after MI. B. Relative KCa3.1 protein expression level. ${ }^{*} \mathrm{P}<0.05$ vs. Sham, ${ }^{* *} \mathrm{P}<0.05$ vs. Day14, ${ }^{* * *} \mathrm{P}<0.05$ vs. Day21, $\mathrm{n}=3$ per time point. C. Relative KCa3.1 mRNA expression level. ${ }^{*} \mathrm{P}<0.05$ VS. Sham, ${ }^{* *} \mathrm{P}<0.05$ VS. $\mathrm{D} 14, \mathrm{n}=3$ per time point.

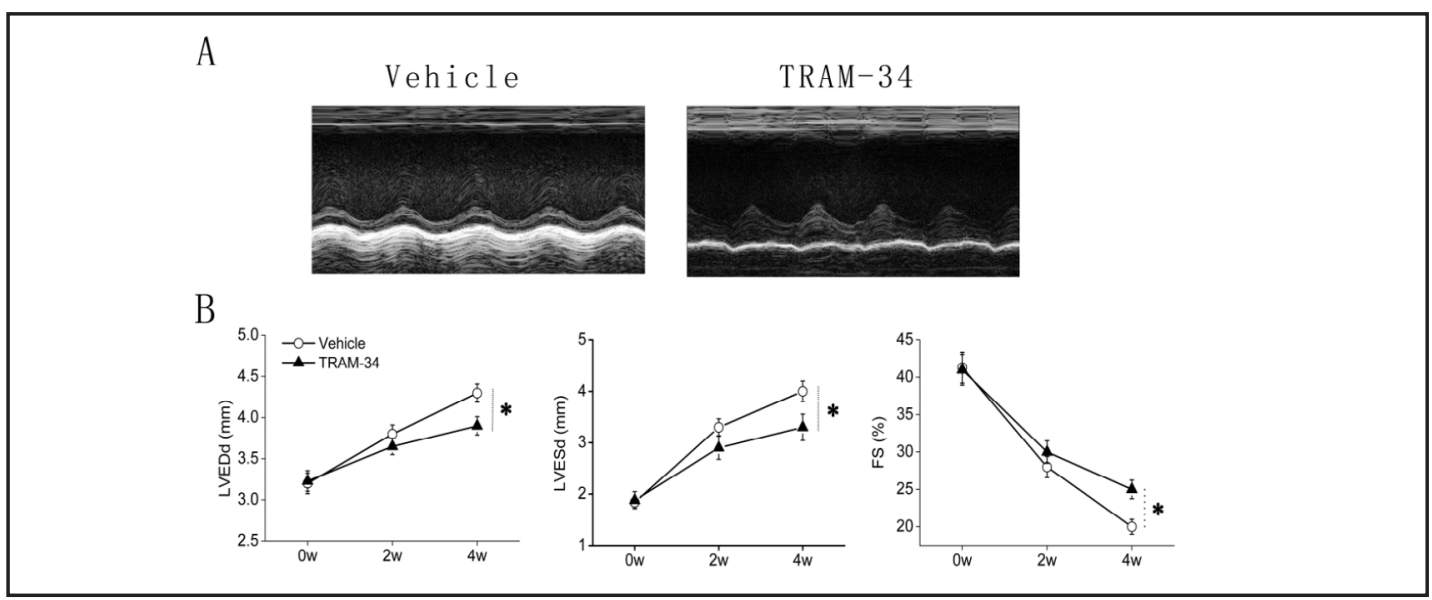

Fig. 2. Pharmacological Blockade of KCa3.1 Channel attenuated post-MI cardiac dysfunction and adverse ventricular remodeling. A. Representative M-mode images from Vehicle and TRAM-34 groups 4 weeks postMI. B. Echocardiographic data show that left ventricular end-diastolic and end-systolic dimensions (LVEDd, LVESd) were smaller and fractional shortening (FS) was greater in TRAM-34 group than in Vehicle group at 4 weeks after MI. ${ }^{*} \mathrm{P}<0.05$ vs. Vehicle group, $\mathrm{n}=5-8$ per time point.

of KCa3.1 protein, which could be inhibited by TRAM-34 (Fig. 4A and 4B). Consistent results were verified by RT-PCR (Fig. 4C).

Blockade of KCa3.1 inhibited CFs proliferation, differentiation, and profibrogenic gene expression

Then study determined to confirm whether blockade of KCa3.1 could regulate Ang II-induced cell proliferation, differentiation, and profibrogenic effects in mouse CFs. The number of CFs was significantly increased by Ang II. When co-cultured with TRAM-34, this effect was antagonized (Fig. 5A). The expression of $\alpha$-SMA was regarded as a marker KARGER 
Fig. 3. Decreased collagen content and lower prevalence of myofibroblasts in mice treated with TRAM-34 compared with mice treated with Vehicle. (A and B). Representative photomicrographs of Masson's trichrome and Sirius red staining and the quantitative analysis at day 28. Scale

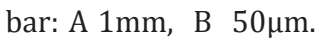
C. Representative photomicrographs of immunohistochemistry using anti- $\alpha$-SMA antibody and quantitative analysis in border zone at day 5. Scale bar: $100 \mu \mathrm{m}$. D. Representative photomicrographs of microvessel as assessed by the number of CD31-positive cells in immunohistochemical staining in infarct zone at day 5. Scale bar: $25 \mu \mathrm{m}$. $(n=5-8$ per group. ${ }^{*} \mathrm{P}<0.05$ VS. Vehicle).

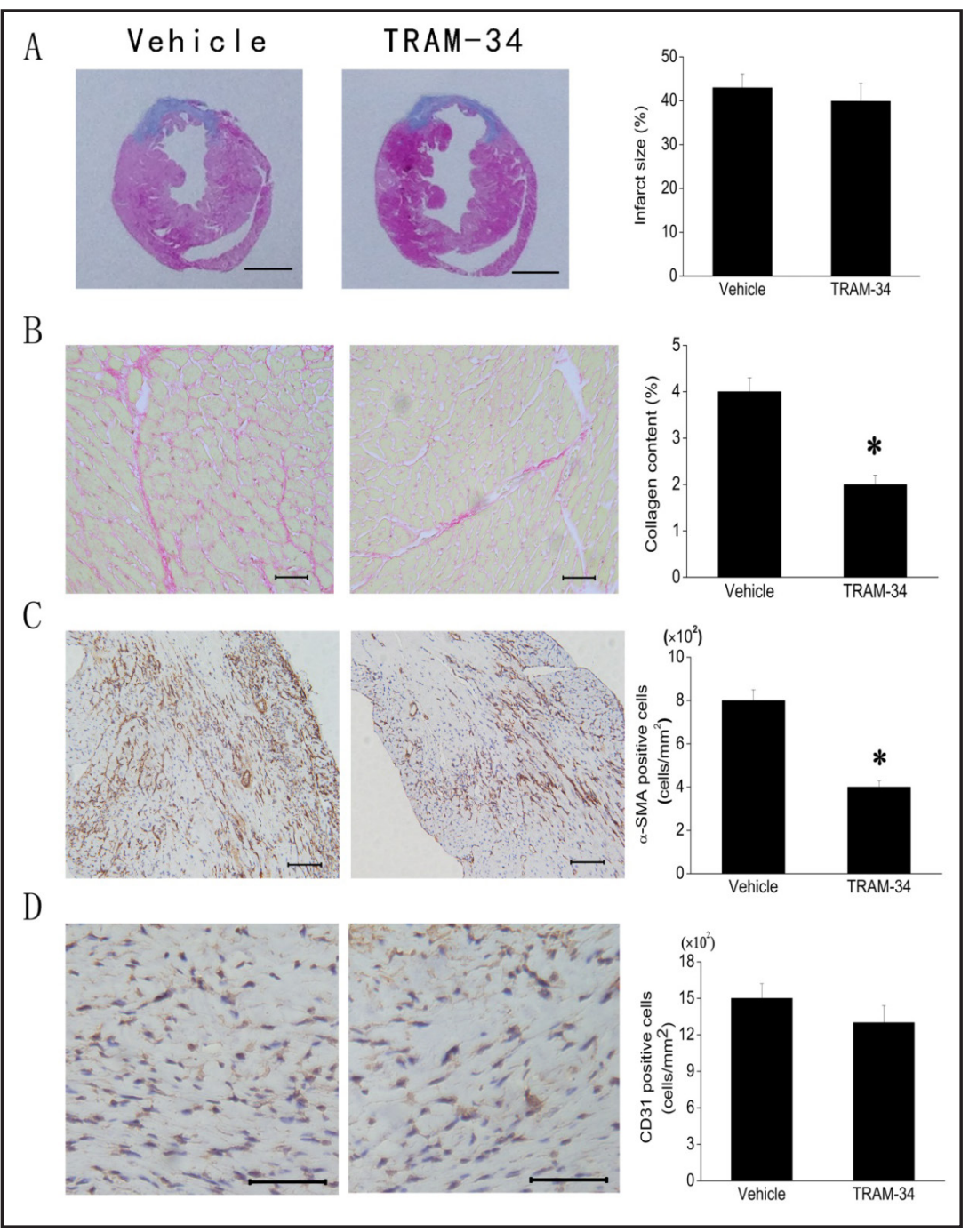

of fibroblasts differentiation to myofibroblasts. The expression of mRNA of $\alpha$-SMA and profibrogenic molecules including transforming growth factor- $\beta 1$ (TGF $\beta 1$ ), procollagens I and III, and connective tissue growth factor(CTGF) was all up regulated by Ang II. These cytokines expression were suppressed by TRAM-34 except for procollagens I (Figs. 5B-5F).

\section{Blockade of KCa3.1 reduced the phosphorylation of AKT and ERK1/2}

To further study whether AKT and ERK-1/2 signalling pathways are regulated by KCa3.1 in CFs, and detected the influence of KCa3.1 blocker on these protein phosphorylation level Therefore, the exposure of CFs to And II resulted in significantly increased AKT and ERK1/2 phosphorylation (Fig. 6). Concurrent exposure to TRAM34 inhibited this effect partially. These results suggest that KCa3.1 may mediate Ang II induced pro-fibrotic effects in CFs through AKT and ERK1/2 signalling pathways.

\section{Discussion}

KCa3.1 was first described by Gardos in erythrocytes in 1958 [20] .Since it was cloned by three groups in 1997 [21-23], its expression and function have been studied extensively. KCa3.1 channels are expressed mainly in hematopoietic derived and gastrointestinal epithelial cells, secretory glands, as well as fibroblasts, vascular endothelial, and vascular smooth muscle cells [9] .Among these different kinds of cells, many participate in the healing 


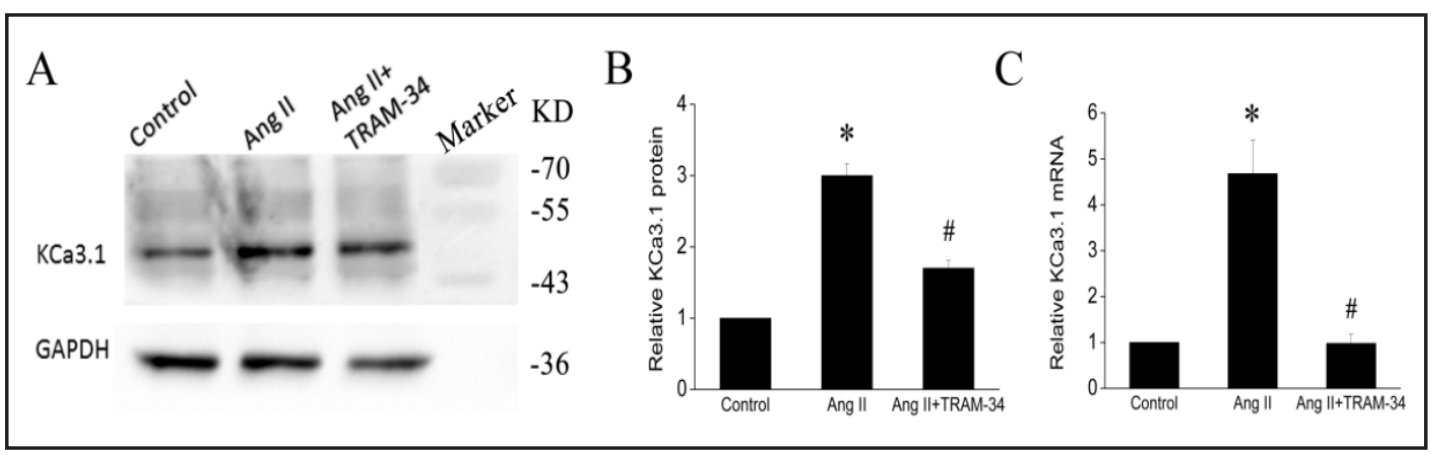

Fig. 4. KCa3.1 was up regulated in Angiotensin II induced cardiac fibroblasts and suppressed by TRAM-34. (A and B). Western blots and relative protein levels of KCa3.1 channels in neonatal mouse cardiac fibroblasts treated with serum free medium, Ang II ( $0.1 \mathrm{uM})$ or Ang II(0.1 uM) plus TRAM-34(1 $\mu \mathrm{M})$. C. Relative KCa3.1 channel mRNA levels with the same treatment with $A .(n=5, * P<0.05$ vs. control, $\# \mathrm{P}<0.05$ vs. And II alone).

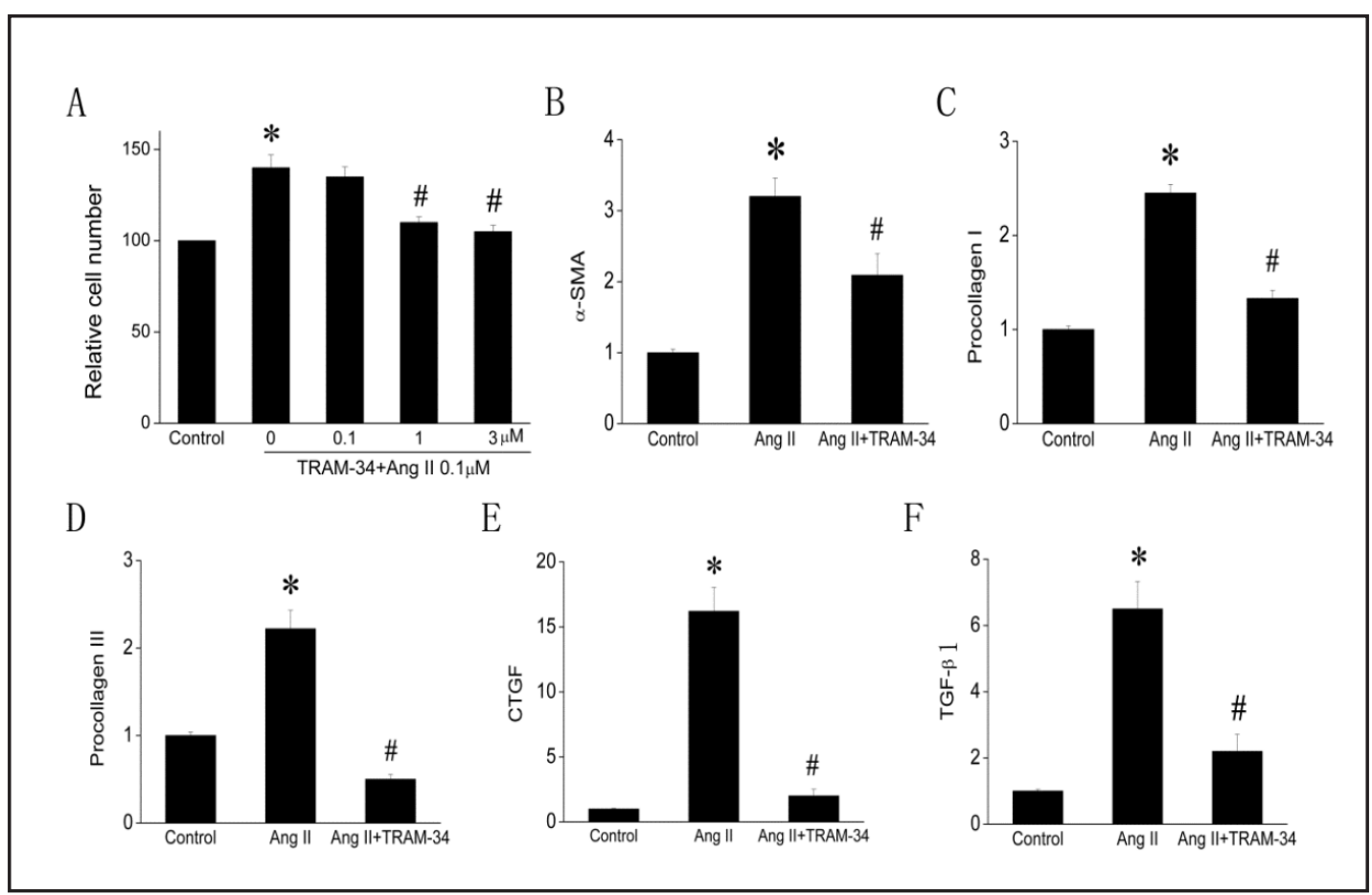

Fig. 5. Blockade of KCa3.1 inhibited cardiac fibroblasts proliferation, differentiation and profibrogenic gene expression. A. Mean values of cell count in CFs treated with serum free medium, Ang II (0.1 $\mu \mathrm{M})$ or Ang II plus $0.1-3 \mu \mathrm{M}$ TRAM-34 for $24 \mathrm{~h}\left(\mathrm{n}=6,{ }^{* *} \mathrm{P}<0.01 \mathrm{vs}\right.$. control, $\# \mathrm{P}<0.01 \mathrm{vs}$. Ang II alone). B-F. Relative mRNA level of $\alpha$-SMA, Procollagen I ,Procollagen III, CTGF, TGF $\beta 1$, in cells treated with Ang II (0.1uM), Ang II plus 1 uM TRAM-34 for $24 \mathrm{~h}$ ( $\mathrm{n}=5, * \mathrm{P}<0.05$ vs. Control, \#P < 0.05 vs. Ang II alone).

process after MI. Thus, the study hypothesized that KCa3.1 may be involved in ventricular remodeling as a pathogenic mechanism.

We proved that selectively blockade of KCa3.1 attenuates LV remodeling after MI. Firstly, we observed a dynamic change of KCa3.1 in the infarct hearts. Results showed an increase of KCa3.1 protein level in the early time of infarct healing and a higher expression level in the later. Though at the end of studying time point we did not see a reduction of KCa3.1, it is obvious that KCa3.1 is associated with both healing and remodeling process after MI. The mRNA expression has a similar change with protein and is consistent with previous study [24] .

\section{KARGER}




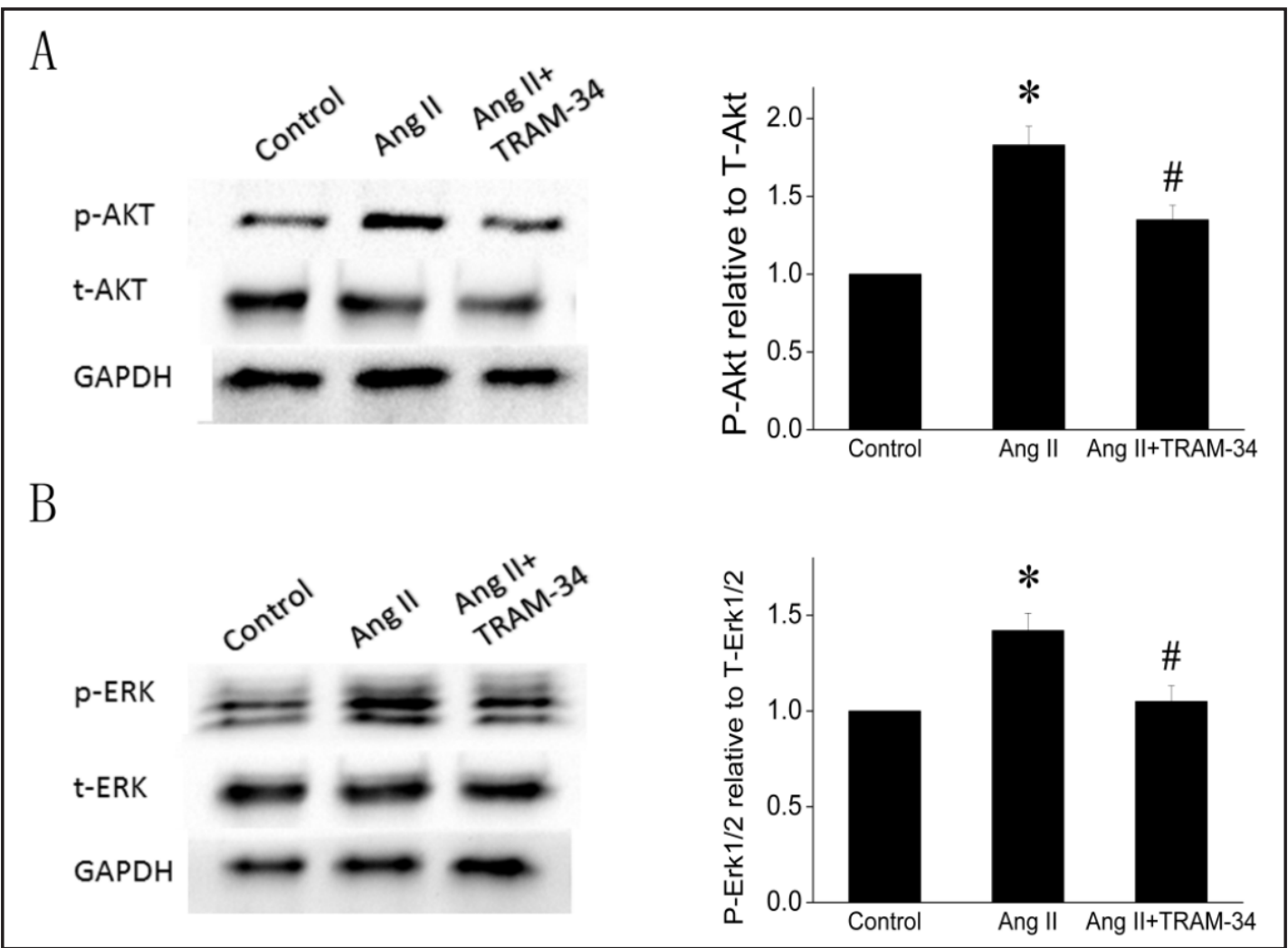

Fig. 6. Blockade of KCa3.1 reduced the phosphoration of AKT and ERK1/2. A. Western blots (left panel) and relative levels (right panel) to total AKT (T-AKT) of phosphorylated AKT (P-AKT) in neonatal mouse cardiac fibroblasts treated with serum free medium, Ang II (0.1uM) or Ang II plus 1 uM TRAM-34 for 30 min. B. Western blots (left panel) and relative levels (right panel) to total ERK1/2 (T-ERK1/2) of phosphorylated ERK1/2 (P-ERK1/2) in neonatal cardiac fibroblasts with the same treatment with A. $\left(\mathrm{n}=5,{ }^{*} \mathrm{P}<0.01\right.$ vs. control, \#P $<0.05$ vs. Ang II alone).

Then a selective KCa3.1 blocker, TRAM-34 was used to study the role of KCa3.1 after MI. TRAM-34 has been applied in various kinds of animal disease models such as renal fibrosis, atherogenesis, and colitis, where its safety and plasma compound concentration after daily intraperitoneal injection were fully tested $[11,25,26]$. So the mice were treated with TRAM343 days before MI surgery used to assure a steady plasma concentration at the whole process of infarct healing and remodeling. Then we tested the number of myofibroblsts and endothelial cells at day 5 after MI when at the peak of their proliferation [27]. The present study found that pharmacological blockade of KCa3.1 improved heart function, reduced $\mathrm{LV}$ dilatation, decreased interstitial fibrosis, and the number of myofibroblasts, indicating a profibrotic role of $\mathrm{KCa} 3.1$ in $\mathrm{LV}$ remodeling after MI. As previous study has proved that Candesartan, a specific antagonist for ang AT1 receptors, can inhibited the elevation of KCa3.1 mRNA expression, we may deduced that KCa3.1 at least partially underlie the beneficial effects of Ang II receptor blockers on myocardial remodeling [24].

Besides the beneficial effects of TRAM-34 after MI, we should also consider the side effects. Immediately following MI, early deposition of collagen in the infarct zone is crucial to maintain the structural integrity of the ventricular wall and preventing aneurysm formation and ventricular rupture. However, during late phase recovery from MI, excessive fibrosis greatly contributed to the progression to heart failure [28]. Thus TRAM-34 treatment which target fibrosis may be harmful to the early deposition of collagen. However, at day 3 after MI, we tested the pro-fibrotic genes and matrix metalloproteinases (MMP) genes expression in infarct zone between two groups and found no difference (date not shown). Besides, during 
the experiment, we did not found a higher rupture and death rate in TRAM-34 group. These evidences showed that TRAM-34 may not influence early phase cardiac restorative fibrosis. The role of KCa3.1 in mouse CFs has not been tested so far. Therefore, to investigate the mechanisms by which TRAM-34 regulates fibrotic remodeling, we focused mainly on CFs which contribute mostly to fibrosis. As we knew, Ang II, greatly upregulated in remodeling heart, and play an important role in stimulating CFs proliferation, differentiation, and expression of profibrogenic cytokines, which all greatly contribute to myocardial remodeling [6]. Then the study demonstrated that the proliferation, differentiation and profibrogenic gene expression of cultured neonatal mouse CFs induced by Ang II could be inhibited by TRAM-34. These data suggest that KCa3.1 is involved in Ang II induced fibroblasts functional changes. This may explain the beneficial effects of KCa3.1 blocker on post MI remodeling.

Activation of ERK1/2 and AKT has been shown to be involved in Ang II induced CFs and play a role in its function $[29,30]$.To demonstrate whether blockade of KCa3.1 has an effect on these signaling cascade, the phosphorylation of AKT and ERK1/2 influenced by TRAM-34 was examined. Results showed a significant reduction of both phosphorylation levels. Taken together, TRAM-34 exhibit its anti-fibrotic effects in Ang II induced CFs through inhibiting the AKT and ERK1/2 signalling pathways.

These in vitro studies provide a possible explanation for the in vivo effects of TRAM-34. But which area it plays roles in is still needs to answer. During recovery from MI, excessive collagen deposition and fibrosis in non-infarct area is viewed as a major contributor to the progression to heart failure[31]. Though the infarct area is less fibrotic in TRAM-34 group, it is still non contractile and its size did not reduce (Fig.3A), which may not account for the improvement of heat function. Thus the less fibrotic non-infarct area induced by TRAM34 may be the key factor. Besides, it is obvious that TRAM-34 is not specific to CFs in this model. Its targets involve other cell types such as vascular, inflammatory, and immune cells (lymphocytes, mast cells and monocyte/macrophages), which play an important role in the pathophysiology of MI[32]. We measured the microvascular density at day 5 and some major inflammation factors mRNA expression at day 3(date not shown) between TRAM-34 and vehicle groups, but found no difference. These suggest that TRAM-34 may not influence the inflammatory and vascular cells.

\section{Conclusion}

The data demonstrated that KCa3.1 blockade has the potential to attenuate ventricular remodeling after MI perhaps through inhibiting the proliferation, differentiation, and profibrogenic effects of CFs mediated by AKT and ERK1/2 signalling pathways. As an orally available KCa3.1 inhibitor Senicapoc have been shown to be well tolerated and produce biological effects in humans, hence there is a great potential that this study may rapidly be transformed to the clinic.

\section{Disclosure Statement}

The authors declare that they have no competing interests.

\section{Acknowledgements}

This work was supported by the National Nature Science Foundation of China (No. U1204801) to Dr. Xian-Pei Wang. The authors would like to thank Research Center of Ion Channelopathy in Wuhan Union Hospital and professor Yi-Mei Du. 


\section{Cellular Physiology Cell Physiol Biochem 2015;36:1305-1315 \begin{tabular}{ll|l} 
and Biochemistry $10.1159 / 000430298$ & $\begin{array}{l}\text { Published onlIne: July } 2015 \text { S. Karger AG, Basel } 2015 \\
\text { www.karger.com/cpb }\end{array}$ \\
\hline
\end{tabular}}

Ju et al.: Blockade of KCa3.1 Attenuates Left Ventricular Remodeling

\section{References}

1 Sutton MGSJ, Sharpe N: Left ventricular remodeling after myocardial infarction : Pathophysiology and therapy. Circulation 2000;101:2981-2988.

-2 Opie LH, Commerford PJ, Gersh BJ, Pfeffer MA: Controversies in ventricular remodelling. Lancet 2006;367:356-367.

-3 Weber KT, Sun Y, Tyagi SC, Cleutjens JP: Collagen network of the myocardium: Function, structural remodeling and regulatory mechanisms. J Mol Cell Cardiol 1994;26:279-292.

-4 Li X, Li F, Chu Y, Wang X, Zhang H, Hu Y, Zhang Y, Wang Z, Wei X, Jian W, Zhang X, Yi F: Nod2 deficiency protects against cardiac remodeling after myocardial infarction in mice. Cell Physiol Biochem 2013;32:1857-1866.

-5 Zannad F, Alla F, Dousset B, Perez A, Pitt B: Limitation of excessive extracellular matrix turnover may contribute to survival benefit of spironolactone therapy in patients with congestive heart failure: Insights from the randomized aldactone evaluation study (rales). Rales investigators. Circulation 2000;102:27002706.

6 Porter KE, Turner NA: Cardiac fibroblasts: At the heart of myocardial remodeling. Pharmacol Ther 2009;123:255-278.

7 Chen W, Frangogiannis NG: Fibroblasts in post-infarction inflammation and cardiac repair. Biochim Biophys Acta 2013;1833:945-953.

8 Mishina H, Watanabe K, Tamaru S, Watanabe Y, Fujioka D, Takahashi S, Suzuki K, Nakamura T, Obata JE, Kawabata K, Yokota Y, Inoue O, Murakami M, Hanasaki K, Kugiyama K: Lack of phospholipase a2 receptor increases susceptibility to cardiac rupture after myocardial infarction. Circ Res 2014;114:493-504.

-9 Wulff H, Castle NA: Therapeutic potential of kca3.1 blockers: Recent advances and promising trends. Exp Rev Clin Pharmacol 2010;3:385-396.

10 Lu FH, Fu SB, Leng X, Zhang X, Dong S, Zhao YJ, Ren H, Li H, Zhong X, Xu CQ, Zhang WH: Role of the calciumsensing receptor in cardiomyocyte apoptosis via the sarcoplasmic reticulum and mitochondrial death pathway in cardiac hypertrophy and heart failure. Cell Physiol Biochem 2013;31:728-743.

11 Grgic I, Kiss E, Kaistha BP, Busch C, Kloss M, Sautter J, Muller A, Kaistha A, Schmidt C, Raman G, Wulff H, Strutz F, Grone HJ, Kohler R, Hoyer J: Renal fibrosis is attenuated by targeted disruption of kca3.1 potassium channels. Proc Natl Acad Sci U S A 2009;106:14518-14523.

12 Huang C, Shen S, Ma Q, Gill A, Pollock CA, Chen XM: Kca3.1 mediates activation of fibroblasts in diabetic renal interstitial fibrosis. Nephrol, dialysis, transplant 2014;29:313-324.

-13 Cruse G, Singh SR, Duffy SM, Doe C, Saunders R, Brightling CE, Bradding P: Functional kca3.1 k+ channels are required for human fibrocyte migration. J Allergy Clin Immunol 2011;128:1303-1309 e1302.

14 Roach KM, Duffy SM, Coward W, Feghali-Bostwick C, Wulff H, Bradding P: The k+ channel kca3.1 as a novel target for idiopathic pulmonary fibrosis. PloS one 2013;8:e85244.

15 White DA, Su Y, Kanellakis P, Kiriazis H, Morand EF, Bucala R, Dart AM, Gao XM, Du XJ: Differential roles of cardiac and leukocyte derived macrophage migration inhibitory factor in inflammatory responses and cardiac remodelling post myocardial infarction. J Mol Cell Cardiol 2014;69:32-42.

-16 Zhao N, Dong Q Fu XX, Du LL, Cheng X, Du YM, Liao YH: Acacetin blocks kv1.3 channels and inhibits human t cell activation. Cell Physiol Biochem 2014;34:1359-1372.

-17 Zhang H, Wu J, Dong H, Khan SA, Chu ML, Tsuda T: Fibulin-2 deficiency attenuates angiotensin ii-induced cardiac hypertrophy by reducing transforming growth factor-beta signalling. Clin Sci 2014;126:275-288.

18 Yan X, Shichita T, Katsumata Y, Matsuhashi T, Ito H, Ito K, Anzai A, Endo J, Tamura Y, Kimura K, Fujita J, Shinmura K, Shen W, Yoshimura A, Fukuda K, Sano M: Deleterious effect of the il-23/il-17a axis and gammadeltat cells on left ventricular remodeling after myocardial infarction. J Am Heart Assoc 2012;1:e004408.

19 Pfeffer JM, Fischer TA, Pfeffer MA: Angiotensin-converting enzyme inhibition and ventricular remodeling after myocardial infarction. Ann Rev Physiol 1995;57:805-826.

20 Gardos G: The function of calcium in the potassium permeability of human erythrocytes. Biochim Biophys Acta 1958;30:653-654.

21 Logsdon NJ, Kang J, Togo JA, Christian EP, Aiyar J: A novel gene, hkca4, encodes the calcium-activated potassium channel in human t lymphocytes. J Biol Chem 1997;272:32723-32726. 


\section{Cellular Physiology Cell Physiol Biochem 2015;36:1305-1315

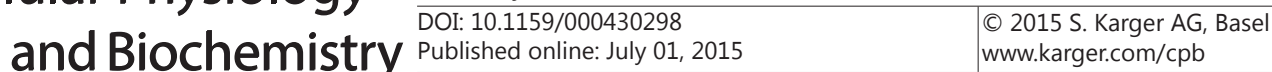 \\ Ju et al.: Blockade of KCa3.1 Attenuates Left Ventricular Remodeling}

-22 Joiner WJ, Wang LY, Tang MD, Kaczmarek LK: Hsk4, a member of a novel subfamily of calcium-activated potassium channels. Proc Natl Acad Sci U S A 1997;94:11013-11018.

23 Ishii TM, Silvia C, Hirschberg B, Bond CT, Adelman JP, Maylie J: A human intermediate conductance calciumactivated potassium channel. Proc Natl Acad Sci U S A 1997;94:11651-11656.

-24 Saito T, Fujiwara Y, Fujiwara R, Hasegawa H, Kibira S, Miura H, Miura M: Role of augmented expression of intermediate-conductance ca2+-activated k+ channels in postischaemic heart. Clin Exp Pharmacol Physiol 2002;29:324-329.

25 Di L, Srivastava S, Zhdanova O, Ding Y, Li Z, Wulff H, Lafaille M, Skolnik EY: Inhibition of the k+ channel kca3.1 ameliorates t cell-mediated colitis. Proc Natl Acad Sci U S A 2010;107:1541-1546.

26 Toyama K, Wulff H, Chandy KG, Azam P, Raman G, Saito T, Fujiwara Y, Mattson DL, Das S, Melvin JE, Pratt PF, Hatoum OA, Gutterman DD, Harder DR, Miura H: The intermediate-conductance calcium-activated potassium channel kca3.1 contributes to atherogenesis in mice and humans. J Clin Invest 2008;118:30253037.

27 Virag JI, Murry CE: Myofibroblast and endothelial cell proliferation during murine myocardial infarct repair. Am J Pathol 2003;163:2433-2440.

28 Sun Y, Weber KT: Infarct scar: A dynamic tissue. Cardiovas Res 2000;46:250-256.

29 Lijnen PJ, van Pelt JF, Fagard RH: Downregulation of manganese superoxide dismutase by angiotensin ii in cardiac fibroblasts of rats: Association with oxidative stress in myocardium. Am J Hypertens 2010;23:11281135.

-30 Zhang W, Chen XF, Huang YJ, Chen QQ, Bao YJ, Zhu W: 2,3,4',5-tetrahydroxystilbene-2-o-beta-d-glucoside inhibits angiotensin ii-induced cardiac fibroblast proliferation via suppression of the reactive oxygen species-extracellular signal-regulated kinase 1/2 pathway. Clin Exp Pharmacol Physiol 2012;39:429-437.

- 31 Borg TK, Ranson WF, Moslehy FA, Caulfield JB: Structural basis of ventricular stiffness. Lab Invest 1981;44:49-54.

-32 Xu CW, Zhang TP, Wang HX, Yang H, Li HH: Chip enhances angiogenesis and restores cardiac function after infarction in transgenic mice. Cell Physiol Biochem 2013;31:199-208. 